UDC: $517.518 .15 / .18: 517.518 .28$

44 (LXX) 2 (2020), 91-106

DOI: $10.37560 /$ matbil $2020091 \mathrm{k}$

\title{
NEW INEQUALITIES FOR LOCAL FRACTIONAL INTEGRALS PERTAINING GENERALIZED STRONGLY CONVEX MAPPINGS
}

\author{
ARTION KASHURI ${ }^{1}$ AND MEHMET ZEKI SARIKAYA ${ }^{2}$
}

\begin{abstract}
In this paper, we first obtain a generalized integral identity for twice local fractional differentiable mappings on fractal sets $\Re^{\alpha}(0<\alpha \leq 1)$ of real line numbers. Then, using twice local fractional differentiable mappings that are in absolute value at certain powers generalized strongly convex, we obtain some new estimates on generalization of trapezium-like inequalities. We also discuss some new special cases which can be deduced from our main results.
\end{abstract}

\section{INTRODUCTION}

Let $\Re, \Re^{+}, \mathbf{Q}, \mathbf{Z}$ and $\mathbf{N}$ be the sets of real numbers, positive real numbers, rational numbers, integers, and positive integers, respectively, and

$$
\mathbf{I}:=\Re \backslash \mathbf{Q} \text { and } \mathbf{N}_{0}:=\mathbf{N} \cup\{0\} .
$$

In order to describe the definition of the local fractional derivative and local fractional integral, recently, one has introduced the following sets, see [8, 21, 24, 27. In this paper we are also motivated by, see [3]-[5]. Recently, the theory of Yang's fractional sets, see [24] and references therein, was introduced as follows.

For $0<\alpha \leq 1$, we have the following $\alpha$-type set of element sets:

(1) The $\alpha$-type set of integers $\mathbf{Z}^{\alpha}$ is defined by

$$
\mathbf{Z}^{\alpha}:=\left\{0^{\alpha}\right\} \cup\left\{ \pm n^{\alpha}: n \in \mathbf{N}\right\}
$$

(2) The $\alpha$-type set of rational numbers $\mathbf{Q}^{\alpha}$ is defined by

$$
\mathbf{Q}^{\alpha}:=\left\{q^{\alpha}: q \in \mathbf{Q}\right\}=\left\{q^{\alpha}=\left(\frac{r}{s}\right)^{\alpha}: r, s \in \mathbf{N}\right\} ;
$$

(3) The $\alpha$-type set of irrational numbers $\mathbf{I}^{\alpha}$ is defined by

$$
\mathbf{I}^{\alpha}:=\left\{i^{\alpha}: i \in \mathbf{I}\right\}=\left\{i^{\alpha} \neq\left(\frac{r}{s}\right)^{\alpha}: r, s \in \mathbf{N}\right\} ;
$$

(4) The $\alpha$-type set of real line numbers $\Re^{\alpha}$ is defined by $\Re^{\alpha}:=\mathbf{Q}^{\alpha} \cup \mathbf{I}^{\alpha}$.

2010 Mathematics Subject Classification. Primary: 26A51; Secondary: 26A33, 26D07, 26D10, $26 \mathrm{D} 15$.

Key words and phrases. Local fractional derivative, Local fractional integral, Fractal space, Generalized Hölder inequality, Generalized power mean inequality, Generalized convex functions. 
Throughout this paper, whenever the $\alpha$-type set $\Re^{\alpha}$ of real line numbers is involved, the $\alpha$ is assumed to be tacitly $0<\alpha \leq 1$. One has also defined two binary operations the addition + and the multiplication - (which is conventionally omitted) on the $\alpha$-type set $\Re^{\alpha}$ of real line numbers as follows. For $a^{\alpha}, b^{\alpha} \in \Re^{\alpha}$, two binary operations the addition + and the multiplication $\cdot$ are defined as

$$
a^{\alpha}+b^{\alpha}:=(a+b)^{\alpha} \text { and } a^{\alpha} \cdot b^{\alpha}=a^{\alpha} b^{\alpha}:=(a b)^{\alpha} .
$$

Then one finds that $\left(\Re^{\alpha},+\right)$ is a commutative group.

For $a^{\alpha}, b^{\alpha}, c^{\alpha} \in \Re^{\alpha}$ the following holds:

(1) $a^{\alpha}+b^{\alpha} \in \Re^{\alpha}$;

(2) $a^{\alpha}+b^{\alpha}=b^{\alpha}+a^{\alpha}$;

(3) $a^{\alpha}+\left(b^{\alpha}+c^{\alpha}\right)=\left(a^{\alpha}+b^{\alpha}\right)+c^{\alpha}$;

(4) $0^{\alpha}$ is the identity for $\left(\Re^{\alpha},+\right)$. For any $a^{\alpha} \in \Re^{\alpha}, a^{\alpha}+0^{\alpha}=0^{\alpha}+a^{\alpha}=a^{\alpha}$;

(5) For each $a^{\alpha} \in \Re^{\alpha},(-a)^{\alpha}$ is the inverse element of $a^{\alpha}$ for $\left(\Re^{\alpha},+\right)$, so we have

$$
a^{\alpha}+(-a)^{\alpha}=(a+(-a))^{\alpha}=0^{\alpha} .
$$

$\left(\Re^{\alpha} \backslash\left\{0^{\alpha}\right\}, \cdot\right)$ is a commutative group.

For $a^{\alpha}, b^{\alpha}, c^{\alpha} \in \Re^{\alpha}$ the following holds:

(1) $a^{\alpha} \cdot b^{\alpha} \in \Re^{\alpha}$;

(2) $a^{\alpha} \cdot b^{\alpha}=b^{\alpha} \cdot a^{\alpha}$

(3) $a^{\alpha} \cdot\left(b^{\alpha} \cdot c^{\alpha}\right)=\left(a^{\alpha} \cdot b^{\alpha}\right) \cdot c^{\alpha}$;

(4) $1^{\alpha}$ is the identity for $\left(\Re^{\alpha}, \cdot\right)$. For any $a^{\alpha} \in \Re^{\alpha}, a^{\alpha} \cdot 1^{\alpha}=1^{\alpha} \cdot a^{\alpha}=a^{\alpha}$;

(5) For each $a^{\alpha} \in \Re^{\alpha} \backslash\left\{0^{\alpha}\right\},\left(\frac{1}{a}\right)^{\alpha}$ is the inverse element of $a^{\alpha}$ for $\left(\Re^{\alpha}, \cdot\right)$, so we have

$$
a^{\alpha} \cdot\left(\frac{1}{a}\right)^{\alpha}=\left(a \cdot\left(\frac{1}{a}\right)\right)^{\alpha}=1^{\alpha} .
$$

Distributive law holds: $a^{\alpha} \cdot\left(b^{\alpha}+c^{\alpha}\right)=a^{\alpha} \cdot b^{\alpha}+a^{\alpha} \cdot c^{\alpha}$.

Furthermore, in [8], we observe some additional properties for $\left(\Re^{\alpha},+, \cdot\right)$ which are stated in the following proposition.

Proposition 1.1. Each of the following statements holds true:

(a) Like the usual real number system $(\Re,+, \cdot),\left(\Re^{\alpha},+, \cdot\right)$ is a field;

(b) The additive identity $0^{\alpha}$ and the multiplicative identity $1^{\alpha}$ are unique;

(c) The additive inverse element $(-a)^{\alpha}$ and the multiplicative inverse element $\left(\frac{1}{a}\right)^{\alpha}$ of element $a^{\alpha}$ are unique;

(d) For each $a^{\alpha} \in \Re^{\alpha}$, its inverse element $(-a)^{\alpha}$ ay be written as $-a^{\alpha}$. For each $b^{\alpha} \in \Re^{\alpha} \backslash\left\{0^{\alpha}\right\}$, its inverse element $\left(\frac{1}{b}\right)^{\alpha}$ ay be written as $\frac{1^{\alpha}}{b^{\alpha}}$ but not as $\frac{1}{b^{\alpha}}$;

(e) If the order $<$ is defined on $\left(\Re^{\alpha},+, \cdot\right)$ as follows: $a^{\alpha}<b^{\alpha}$ in $\Re^{\alpha}$ if and only if $a<b$ in $\Re$, then $\left(\Re^{\alpha},+, \cdot,<\right)$ is an ordered field like $(\Re,+, \cdot,<)$. 
In order to introduce the local fractional calculus on $\Re^{\alpha}$, we begin with the concept of the local fractional continuity as in Definition 1.1

Definition 1.1. A non-differentiable function $f: \Re \rightarrow \Re^{\alpha}, x \rightarrow f(x)$, is said to be local fractional continuous at $x_{0}$ if for any $\epsilon \in \Re^{+}$, there exists $\delta \in \Re^{+}$such that

$$
\left|f(x)-f\left(x_{0}\right)\right|<\epsilon^{\alpha}
$$

holds for $\left|x-x_{0}\right|<\delta$. If a function $f$ is local continuous on the interval $(a, b)$, we denote $f \in C_{\alpha}(a, b)$.

Among several attempts to have defined local fractional derivative and local fractional integral, see ([23], Section 2.1), we choose to recall the following definitions of local fractional calculus, see [9, 23, 24].

Definition 1.2. The local fractional derivative of $f(x)$ of order $\alpha$ at $x=x_{0}$ is defined by

$$
f^{(\alpha)}\left(x_{0}\right)={ }_{x_{0}} D_{x}^{\alpha} f(x)=\left.\frac{d^{\alpha} f(x)}{d x^{\alpha}}\right|_{x=x_{0}}=\lim _{x \rightarrow x_{0}} \frac{\Delta^{\alpha}\left(f(x)-f\left(x_{0}\right)\right)}{\left(x-x_{0}\right)^{\alpha}},
$$

where $\Delta^{\alpha}\left(f(x)-f\left(x_{0}\right)\right) \cong \Gamma(1+\alpha)\left(f(x)-f\left(x_{0}\right)\right)$ and $\Gamma$ is the familiar gamma function, see ([20], Section 1.1).

Let $f^{(\alpha)}(x)=D_{x}^{\alpha} f(x)$. If there exists $f^{(k+1) \alpha}(x)=\underbrace{D_{x}^{\alpha} \ldots D_{x}^{\alpha}}_{k+1 \text { times }} f(x)$ for any $x \in I \subseteq \Re$, then we denote $f \in D_{(k+1) \alpha}(I)$, where $k \in \mathbf{N}_{0}$.

Remark 1.1: It is found, (see [24] and references therein), in this expression, $\alpha$ is precisely the Hölder exponent of function defined Cantor's set. That is to say, $\left[d\left(x-x_{0}\right)\right]^{\alpha}$ which is a fractal span, is a fractal geometrical meaning.

Definition 1.3. Let $f \in C_{\alpha}[a, b]$. Also let $P=\left\{t_{0}, \ldots, t_{N}\right\},(N \in \mathbf{N})$ be a partition of the interval $[a, b]$ which satisfies $a=t_{0}<t_{1}<\cdots<t_{N-1}<t_{N}=b$. Further, for this partition $P$, let $\Delta t:=\max _{0 \leq j \leq N-1} \Delta t_{j}$, where $\Delta t_{j}:=t_{j+1}-t_{j}$ and $j=0, \ldots, N-1$. Then the local fractional integral of $f$ on the interval $[a, b]$ of order $\alpha$ (denoted by $\left.{ }_{a} I_{b}^{(\alpha)} f\right)$ is defined by

$$
{ }_{a} I_{b}^{(\alpha)} f(t)=\frac{1}{\Gamma(1+\alpha)} \int_{a}^{b} f(t)(d t)^{\alpha}:=\frac{1}{\Gamma(1+\alpha)} \lim _{\Delta t \rightarrow 0} \sum_{j=0}^{N-1} f\left(t_{j}\right)\left(\Delta t_{j}\right)^{\alpha},
$$

provided the limit exists (in fact, this limit exists if $f \in C_{\alpha}[a, b]$ ).

Here, it follows that ${ }_{a} I_{b}^{(\alpha)} f=0$ if $a=b$ and ${ }_{a} I_{b}^{(\alpha)} f=-{ }_{b} I_{a}^{(\alpha)} f$ if $a<b$. If ${ }_{a} I_{x}^{(\alpha)} g$ exists for any $x \in[a, b]$ and a function $g:[a, b] \rightarrow \Re^{\alpha}$, then we denote $g \in I_{x}^{(\alpha)}[a, b]$.

We present some of the features related to the local fractional calculus that will be required for our main results, see [24].

Lemma 1. The following identities hold true: 
(1) (Local fractional derivative of $x^{k \alpha}$ ).

$$
\frac{d^{\alpha} x^{k \alpha}}{d x^{\alpha}}=\frac{\Gamma(1+k \alpha)}{\Gamma(1+(k-1) \alpha)} x^{(k-1) \alpha} .
$$

(2) (Local fractional integration is anti-differentiation). Suppose that $f(x)=g^{(\alpha)}(x) \in C_{\alpha}[a, b]$. Then we have

$$
{ }_{a} I_{b}^{(\alpha)} f(x)=g(b)-g(a) .
$$

(3) (Local fractional integration by parts).

Suppose that $f(x), g(x) \in D_{\alpha}[a, b]$ and $f^{(\alpha)}(x), g^{(\alpha)}(x) \in C_{\alpha}[a, b]$. Then we have

$$
{ }_{a} I_{b}^{(\alpha)} f(x) g^{(\alpha)}(x)=\left.f(x) g(x)\right|_{a} ^{b}-{ }_{a} I_{b}^{(\alpha)} f^{(\alpha)}(x) g(x) .
$$

(4) (Local fractional definite integrals of $x^{k \alpha}$ ).

$$
\frac{1}{\Gamma(1+\alpha)} \int_{a}^{b} x^{k \alpha}(d x)^{\alpha}=\frac{\Gamma(1+k \alpha)}{\Gamma(1+(k+1) \alpha)}\left(b^{(k+1) \alpha}-a^{(k+1) \alpha}\right), \quad k \in \Re .
$$

For further details on local fractional calculus, one ay refer to, see [22]-[26].

Definition 1.4. ([21]) A function $f: I \subset \Re \rightarrow \Re$ is said to be convex on $I$, if

$$
f(t x+(1-t) y) \leq t f(x)+(1-t) f(y)
$$

holds for every $x, y \in I$ and $t \in[0,1]$.

Definition 1.5. ([11]) A function $f:[0, b] \rightarrow \Re$ is called $m$-convex with $m \in[0,1]$, if for any $x, y \in[0, b]$ and $t \in[0,1]$ we have

$$
f(t x+m(1-t) y) \leq t f(x)+m(1-t) f(y) .
$$

Definition 1.6. (([1]) A function $f: I \subset \Re \rightarrow \Re$ is called strongly convex with modulus $c \in \Re^{+}$, if

$$
f(t x+(1-t) y) \leq t f(x)+(1-t) f(y)-c t(1-t)(x-y)^{2}
$$

holds for every $x, y \in I$ and $t \in[0,1]$.

Strongly convex functions have been introduced by Polyak, see [11 and references therein. Since strong convexity is a strengthening of the notion of convexity, some properties of strongly convex functions are just stronger versions of known properties of convex functions. Strongly convex functions have been used for proving the convergence of a gradient type algorithm for minimizing a function. They play an important role in optimization theory and atheatical economics.

Definition 1.7. ([1] ) A function $f: I \subset \Re \rightarrow \Re$ is called strongly $m$-convex with $m \in[0,1]$ and modulus $c \in \Re^{+}$, if

$$
f(t x+m(1-t) y) \leq t f(x)+m(1-t) f(y)-\operatorname{cmt}(1-t)(x-y)^{2}
$$

holds for every $x, y \in I$ and $t \in[0,1]$.

Remark 1.2: Any strongly $m$-convex function is, in particular, $m$-convex. However, there are $m$-convex functions, which are not strongly $m$-convex with modulus $c$, for some $c \in \Re^{+}$, (see [11], Example 1.8). 
The following inequality, named Hermite-Hadamard inequality, is one of the most famous inequalities in the literature for convex functions.

Theorem 1. Let $f: I \subset \Re \rightarrow \Re$ be a convex function on $I$ and $a, b \in I$ with $a<b$. Then the following inequality holds:

$$
f\left(\frac{a+b}{2}\right) \leq \frac{1}{b-a} \int_{a}^{b} f(x) d x \leq \frac{f(a)+f(b)}{2} .
$$

Mo et al. in [15, introduced the following generalized convex function.

Definition 1.8. Let $f: I \subset \Re \rightarrow \Re^{\alpha}$ be a function. For any $x_{1}, x_{2} \in I$ and $\lambda \in[0,1]$, if the following inequality

$$
f\left(\lambda x_{1}+(1-\lambda) x_{2}\right) \leq \lambda^{\alpha} f\left(x_{1}\right)+(1-\lambda)^{\alpha} f\left(x_{2}\right)
$$

holds, then $f$ is called a generalized convex function on $I$.

Here are two basic examples of generalized convex functions:

(1) $f(x)=x^{\alpha p}$, where $x \geq 0$ and $p>1$;

(2) $g(x)=E_{\alpha}\left(x^{\alpha}\right), x \in \Re$, where $E_{\alpha}\left(x^{\alpha}\right):=\sum_{k=0}^{\infty} \frac{x^{\alpha k}}{\Gamma(1+k \alpha)}$ is the MittagLeffler function.

Recently the fractal theory has received a significant attention, see [1]-[19], [22][27. Mo et al. in [15], proved the following analogue of the Hermite-Hadamard inequality (1.1) for generalized convex functions.

Theorem 2. Let $f:[a, b] \rightarrow \Re^{\alpha}$ be a generalized convex function with $a<b$. Then, for all $x \in[a, b]$, the following inequality holds:

$$
f\left(\frac{a+b}{2}\right) \leq \frac{\Gamma(1+\alpha)}{(b-a)^{\alpha}}{ }_{a} I_{b}^{(\alpha)} f(x) \leq \frac{f(a)+f(b)}{2^{\alpha}} .
$$

Remark 1.3: The double inequality $(1.2)$ is known in the literature as generalized Hermite-Hadamard integral inequality for generalized convex functions. Some of the classical inequalities for means can be derived from 1.2 with appropriate selections of the mapping $f$. Both inequalities in 1.1 and 1.2 hold in the reverse direction if $f$ is concave and generalized concave, respectively. For some more results which generalize, improve, and extend the inequality 1.2 , one ay refer to the recent papers, see [7, 12, 14, [16]-18] and references therein.

An analogue in the fractal set $\Re^{\alpha}$ of the classical Hölder inequality has been established by Yang in [24], which is asserted by the following lemma.

Lemma 2. Let $f, g \in C_{\alpha}[a, b]$ with $p^{-1}+q^{-1}=1$, where $p, q>1$. Then, we have

$$
\frac{1}{\Gamma(1+\alpha)} \int_{a}^{b}|f(x) g(x)|(d x)^{\alpha} \leq
$$




$$
\leq\left(\frac{1}{\Gamma(1+\alpha)} \int_{a}^{b}|f(x)|^{p}(d x)^{\alpha}\right)^{\frac{1}{p}}\left(\frac{1}{\Gamma(1+\alpha)} \int_{a}^{b}|g(x)|^{q}(d x)^{\alpha}\right)^{\frac{1}{q}} .
$$

Theorem 3. (Generalized Ostrowski inequality) Let $I \subset \Re$ be an interval, $f$ : $I^{0} \subset \Re \rightarrow \Re^{\alpha}\left(I^{0}\right.$ is the interior of $\left.I\right)$ such that $f \in D_{\alpha}\left(I^{0}\right)$, and $f^{(\alpha)} \in C_{\alpha}[a, b]$ for $a, b \in I^{0}$ with $a<b$. Then, for all $x \in[a, b]$, the following inequality holds:

$$
\left|f(x)-\frac{\Gamma(1+\alpha)}{(b-a)^{\alpha}}{ }_{a} I_{b}^{(\alpha)} f(t)\right| \leq 2^{\alpha} \frac{\Gamma(1+\alpha)}{\Gamma(1+2 \alpha)}\left[\frac{1}{4^{\alpha}}+\left(\frac{x-\frac{a+b}{2}}{b-a}\right)^{2 \alpha}\right](b-a)^{\alpha}\left\|f^{(\alpha)}\right\|_{\infty}
$$

Motivated by the above literatures, in the next section, we first introduce the notion of generalized strongly convex mappings and after that we will obtain a generalized integral identity for twice local differentiable mappings on fractal sets $\Re^{\alpha}(0<\alpha \leq 1)$ of real line numbers. Also, we use this identity to obtain some new estimates on generalization of trapezium-like inequalities for twice local fractional differentiable mappings that are in absolute value at certain powers generalized strongly convex. We will discuss some new special cases which can be deduced from our main results.

\section{MAIN RESUlts}

The following definitions will be used in the sequel.

Definition 2.1. ([10]) For $0<\alpha \leq 1$ and $x \in \Re^{+}$, the local gamma function is defined by

$$
\Gamma_{\alpha}(x)=\frac{1}{\alpha !} \int_{0}^{\infty} E_{\alpha}\left(-t^{\alpha}\right) t^{(x-1) \alpha}(d t)^{\alpha} .
$$

For $\alpha=1,(2.1)$ gives integral representation of classical Euler gamma function $\Gamma(x)$. So in this case, $\Gamma_{\alpha}(x)=\Gamma(x)$.

Also, the following relations holds for local gamma function:

(1) $\Gamma_{\alpha}(x+1)=(\alpha !) x \Gamma_{\alpha}(x)$, for $x \in \Re^{+}$;

(2) $\Gamma_{\alpha}(n+1)=(\alpha \text { ! })^{n} n$ !, for $n \in \mathbf{N}$.

Definition 2.2. ([10]) For $0<\alpha \leq 1$ and $x, y \in \Re^{+}$, the local beta function with two parameters $x$ and $y$ is defined as

$$
B_{\alpha}(x, y)=\int_{0}^{1} t^{(x-1) \alpha}(1-t)^{(y-1) \alpha}(d t)^{\alpha} .
$$

For $\alpha=1$, 2.2 gives integral representation of classical Euler beta function $\beta(x, y)$. So, in this case, $B_{\alpha}(x, y)=\beta(x, y)$.

Theorem 4. ([10]) Let $x, y \in \Re^{+}$, then for local gamma and local beta function the following equality holds:

$$
B_{\alpha}(x, y)=\frac{\Gamma_{\alpha}(x) \Gamma_{\alpha}(y)}{\Gamma_{\alpha}(x+y)} .
$$


Recently, Anastassiou et al. in [6], introduced a new class, called generalized strongly $m$-convex, as follows.

Definition 2.3. A function $f: I \subset \Re \rightarrow \Re^{\alpha}$ is called generalized strongly mconvex with $m \in[0,1]$ and modulus $c \in \Re^{+}$, if

$f\left(\lambda x_{1}+m(1-\lambda) x_{2}\right) \leq \lambda^{\alpha} f\left(x_{1}\right)+m^{\alpha}(1-\lambda)^{\alpha} f\left(x_{2}\right)-(c m)^{\alpha} \lambda^{\alpha}(1-\lambda)^{\alpha}\left(x_{1}-x_{2}\right)^{2 \alpha}$

holds for any $x_{1}, x_{2} \in I$ and $\lambda \in[0,1]$.

Remark 2.1: In Definition 2.3 if we choose $\alpha=1$, then we get Definition 1.7 . These mean that, any generalized strongly $m$-convex mapping is, in particular, strongly $m$-convex. Moreover, if $f$ is generalized strongly $m$-convex with modulus $c$, then $f$ is generalized strongly $m$-convex with modulus $k$, for any constant $0<$ $k<c$.

As special case of Definition 2.3 for $m=1$, we obtain the following definition.

Definition 2.4. A function $f: I \subset \Re \rightarrow \Re^{\alpha}$ is called generalized strongly convex with modulus $c \in \Re^{+}$, if

$$
f\left(\lambda x_{1}+(1-\lambda) x_{2}\right) \leq \lambda^{\alpha} f\left(x_{1}\right)+(1-\lambda)^{\alpha} f\left(x_{2}\right)-c^{\alpha} \lambda^{\alpha}(1-\lambda)^{\alpha}\left(x_{1}-x_{2}\right)^{2 \alpha}
$$

holds for any $x_{1}, x_{2} \in I$ and $\lambda \in[0,1]$.

For establishing our main results regarding some new estimates on generalization of trapezium-like integral inequalities on fractal sets $\Re^{\alpha}(0<\alpha \leq 1)$, we need the following lemma.

Lemma 3. Let $I \subset \Re$ be an interval, $f: I^{0} \subset \Re \rightarrow \Re^{\alpha}\left(I^{0}\right.$ is the interior of $\left.I\right)$ such that $f^{(\alpha)} \in D_{\alpha}\left(I^{0}\right)$ and $f^{(2 \alpha)} \in C_{\alpha}[a, b]$ for $a, b \in I^{0}$ with $a<b$. Then, for all $x \in[a, b]$, the following identity holds:

$$
\begin{gathered}
\frac{1}{(b-a)^{\alpha}}{ }_{a} I_{b}^{(\alpha)} f(t)-\frac{1}{4^{\alpha} \Gamma^{2}(1+\alpha)}\left[f\left(\frac{3 a+b}{4}\right)+f\left(\frac{a+b}{2}\right)+f\left(\frac{a+3 b}{4}\right)+f(b)\right] \\
+\frac{(b-a)^{\alpha}}{{ }^{2 \alpha} \Gamma(1+\alpha) \Gamma(1+2 \alpha)}\left[f^{(\alpha)}\left(\frac{3 a+b}{4}\right)+f^{(\alpha)}\left(\frac{a+b}{2}\right)+f^{(\alpha)}\left(\frac{a+3 b}{4}\right)+f^{(\alpha)}(b)\right] \\
=\frac{1}{\Gamma(1+\alpha) \Gamma(1+2 \alpha)(b-a)^{\alpha}} \int_{a}^{b} p(t) f^{(2 \alpha)}(t)(d t)^{\alpha},
\end{gathered}
$$

where

$$
p(t)= \begin{cases}(t-a)^{2 \alpha}, & t \in\left[a, \frac{3 a+b}{4}\right) \\ \left(t-\frac{3 a+b}{4}\right)^{2 \alpha}, & t \in\left[\frac{3 a+b}{4}, \frac{a+b}{2}\right) \\ \left(t-\frac{a+b}{2}\right)^{2 \alpha}, & t \in\left[\frac{a+b}{2}, \frac{a+3 b}{4}\right) \\ \left(t-\frac{a+3 b}{4}\right)^{2 \alpha}, & t \in\left[\frac{a+3 b}{4}, b\right]\end{cases}
$$

We denote

$$
A_{f}^{(\alpha)}(a, b)=\frac{1}{\Gamma(1+\alpha) \Gamma(1+2 \alpha)(b-a)^{\alpha}} \int_{a}^{b} p(t) f^{(2 \alpha)}(t)(d t)^{\alpha} .
$$


Proof. Using twice the local fractional integration by parts and changing the variables in (2.7), we have

$$
\begin{aligned}
& A_{f}^{(\alpha)}(a, b)=\frac{1}{\Gamma(1+\alpha) \Gamma(1+2 \alpha)(b-a)^{\alpha}} \\
& \times\left[\int_{a}^{\frac{3 a+b}{4}}(t-a)^{2 \alpha} f^{(2 \alpha)}(t)(d t)^{\alpha}+\int_{\frac{3 a+b}{4}}^{\frac{a+b}{2}}\left(t-\frac{3 a+b}{4}\right)^{2 \alpha} f^{(2 \alpha)}(t)(d t)^{\alpha}\right. \\
& \left.+\int_{\frac{a+b}{2}}^{\frac{a+3 b}{4}}\left(t-\frac{a+b}{2}\right)^{2 \alpha} f^{(2 \alpha)}(t)(d t)^{\alpha}+\int_{\frac{a+3 b}{4}}^{b}\left(t-\frac{a+3 b}{4}\right)^{2 \alpha} f^{(2 \alpha)}(t)(d t)^{\alpha}\right] \\
& =\frac{(b-a)^{2 \alpha}}{4^{3 \alpha} \Gamma(1+\alpha) \Gamma(1+2 \alpha)} \\
& \times \int_{0}^{1} t^{2 \alpha}\left[f^{(2 \alpha)}\left(\left(\frac{3 a+b}{4}\right) t+(1-t) a\right)+f^{(2 \alpha)}\left(\left(\frac{a+b}{2}\right) t+\left(\frac{3 a+b}{4}\right)(1-t)\right)\right. \\
& \left.+f^{(2 \alpha)}\left(\left(\frac{a+3 b}{4}\right) t+\left(\frac{a+b}{2}\right)(1-t)\right)+f^{(2 \alpha)}\left(b t+\left(\frac{a+3 b}{4}\right)(1-t)\right)\right](d t)^{\alpha} \\
& =\frac{(b-a)^{2 \alpha}}{4^{3 \alpha} \Gamma(1+\alpha) \Gamma(1+2 \alpha)} \times\left\{\left.\frac{4^{\alpha} t^{2 \alpha}}{(b-a)^{\alpha}} f^{(\alpha)}\left(\left(\frac{3 a+b}{4}\right) t+(1-t) a\right)\right|_{0} ^{1}\right. \\
& -\frac{4^{\alpha} \Gamma(1+2 \alpha)}{\Gamma(1+\alpha)(b-a)^{\alpha}} \int_{0}^{1} t^{\alpha} f^{(\alpha)}\left(\left(\frac{3 a+b}{4}\right) t+(1-t) a\right)(d t)^{\alpha} \\
& +\left.\frac{4^{\alpha} t^{2 \alpha}}{(b-a)^{\alpha}} f^{(\alpha)}\left(\left(\frac{a+b}{2}\right) t+\left(\frac{3 a+b}{4}\right)(1-t)\right)\right|_{0} ^{1} \\
& -\frac{4^{\alpha} \Gamma(1+2 \alpha)}{\Gamma(1+\alpha)(b-a)^{\alpha}} \int_{0}^{1} t^{\alpha} f^{(\alpha)}\left(\left(\frac{a+b}{2}\right) t+\left(\frac{3 a+b}{4}\right)(1-t)\right)(d t)^{\alpha} \\
& +\left.\frac{4^{\alpha} t^{2 \alpha}}{(b-a)^{\alpha}} f^{(\alpha)}\left(\left(\frac{a+3 b}{4}\right) t+\left(\frac{a+b}{2}\right)(1-t)\right)\right|_{0} ^{1} \\
& -\frac{4^{\alpha} \Gamma(1+2 \alpha)}{\Gamma(1+\alpha)(b-a)^{\alpha}} \int_{0}^{1} t^{\alpha} f^{(\alpha)}\left(\left(\frac{a+3 b}{4}\right) t+\left(\frac{a+b}{2}\right)(1-t)\right)(d t)^{\alpha} \\
& +\left.\frac{4^{\alpha} t^{2 \alpha}}{(b-a)^{\alpha}} f^{(\alpha)}\left(b t+\left(\frac{a+3 b}{4}\right)(1-t)\right)\right|_{0} ^{1} \\
& \left.-\frac{4^{\alpha} \Gamma(1+2 \alpha)}{\Gamma(1+\alpha)(b-a)^{\alpha}} \int_{0}^{1} t^{\alpha} f^{(\alpha)}\left(b t+\left(\frac{a+3 b}{4}\right)(1-t)\right)(d t)^{\alpha}\right\} \\
& =\frac{(b-a)^{2 \alpha}}{4^{3 \alpha} \Gamma(1+\alpha) \Gamma(1+2 \alpha)} \times\left\{\frac{4^{\alpha}}{(b-a)^{\alpha}} f^{(\alpha)}\left(\frac{3 a+b}{4}\right)-\frac{4^{\alpha} \Gamma(1+2 \alpha)}{\Gamma(1+\alpha)(b-a)^{\alpha}}\right. \\
& \times\left[\left.\frac{4^{\alpha} t^{\alpha}}{(b-a)^{\alpha}} f\left(\left(\frac{3 a+b}{4}\right) t+(1-t) a\right)\right|_{0} ^{1}\right. \\
& \left.-\frac{4^{\alpha} \Gamma(1+\alpha)}{(b-a)^{\alpha}} \int_{0}^{1} f\left(\left(\frac{3 a+b}{4}\right) t+(1-t) a\right)(d t)^{\alpha}\right] \\
& +\frac{4^{\alpha}}{(b-a)^{\alpha}} f^{(\alpha)}\left(\frac{a+b}{2}\right)-\frac{4^{\alpha} \Gamma(1+2 \alpha)}{\Gamma(1+\alpha)(b-a)^{\alpha}}
\end{aligned}
$$




$$
\begin{gathered}
\times\left[\left.\frac{4^{\alpha} t^{\alpha}}{(b-a)^{\alpha}} f\left(\left(\frac{a+b}{2}\right) t+\left(\frac{3 a+b}{4}\right)(1-t)\right)\right|_{0} ^{1}\right. \\
\left.-\frac{4^{\alpha} \Gamma(1+\alpha)}{(b-a)^{\alpha}} \int_{0}^{1} f\left(\left(\frac{a+b}{2}\right) t+\left(\frac{3 a+b}{4}\right)(1-t)\right)(d t)^{\alpha}\right] \\
+\frac{4^{\alpha}}{(b-a)^{\alpha}} f^{(\alpha)}\left(\frac{a+3 b}{4}\right)-\frac{4^{\alpha} \Gamma(1+2 \alpha)}{\Gamma(1+\alpha)(b-a)^{\alpha}} \\
\times\left[\left.\frac{4^{\alpha} t^{\alpha}}{(b-a)^{\alpha}} f\left(\left(\frac{a+3 b}{4}\right) t+\left(\frac{a+b}{2}\right)(1-t)\right)\right|_{0} ^{1}\right. \\
\left.-\frac{4^{\alpha} \Gamma(1+\alpha)}{(b-a)^{\alpha}} \int_{0}^{1} f\left(\left(\frac{a+3 b}{4}\right) t+\left(\frac{a+b}{2}\right)(1-t)\right)(d t)^{\alpha}\right] \\
+\frac{4^{\alpha}}{(b-a)^{\alpha}} f^{(\alpha)}(b)-\frac{4^{\alpha} \Gamma(1+2 \alpha)}{\Gamma(1+\alpha)(b-a)^{\alpha}} \\
\times\left[\left.\frac{4^{\alpha} t^{\alpha}}{(b-a)^{\alpha}} f\left(b t+\left(\frac{a+3 b}{4}\right)(1-t)\right)\right|_{0} ^{1}\right. \\
\left.\left.-\frac{4^{\alpha} \Gamma(1+\alpha)}{(b-a)^{\alpha}} \int_{0}^{1} f\left(b t+\left(\frac{a+3 b}{4}\right)(1-t)\right)(d t)^{\alpha}\right]\right\} \\
=\frac{1}{(b-a)^{\alpha}} I_{b}^{(\alpha)} f(t)-\frac{1}{4^{\alpha} \Gamma^{2}(1+\alpha)}\left[f\left(\frac{3 a+b}{4}\right)+f\left(\frac{a+b}{2}\right)+f\left(\frac{a+3 b}{4}\right)+f(b)\right] \\
+\frac{(b-a)^{\alpha}}{4^{2 \alpha} \Gamma(1+\alpha) \Gamma(1+2 \alpha)}\left[f^{(\alpha)}\left(\frac{3 a+b}{4}\right)+f^{(\alpha)}\left(\frac{a+b}{2}\right)+f^{(\alpha)}\left(\frac{a+3 b}{4}\right)+f^{(\alpha)}(b)\right] .
\end{gathered}
$$

This completes the proof of the lemma.

Using Lemma 3, we now state the following theorems for twice local fractional differentiable mappings that are in absolute value at certain powers generalized strongly convex.

Theorem 5. Suppose that the assumptions of Lemma 3 are satisfied. If $\left|f^{(2 \alpha)}\right|^{q}$ is generalized strongly convex, then for $p, q>1$, where $p^{-1}+q^{-1}=1$ and $c \in \Re^{+}$, the following inequality holds:

$$
\begin{gathered}
\left|A_{f}^{(\alpha)}(a, b)\right| \leq \frac{(b-a)^{2 \alpha}}{4^{3 \alpha} \Gamma(1+2 \alpha)} \sqrt[p]{\frac{\Gamma(1+2 p \alpha)}{\Gamma(1+(2 p+1) \alpha)}} \\
\times\left\{\sqrt[q]{\frac{\Gamma(1+\alpha)}{\Gamma(1+2 \alpha)}\left(\left|f^{(2 \alpha)}\left(\frac{3 a+b}{4}\right)\right|^{q}+\left|f^{(2 \alpha)}(a)\right|^{q}\right)-c^{\alpha}\left(\frac{b-a}{4}\right)^{2 \alpha} \frac{B_{\alpha}(2,2)}{\Gamma(1+\alpha)}}\right. \\
+\left[\frac{\Gamma(1+\alpha)}{\Gamma(1+2 \alpha)}\left(\left|f^{(2 \alpha)}\left(\frac{a+b}{2}\right)\right|^{q}+\left|f^{(2 \alpha)}\left(\frac{3 a+b}{4}\right)\right|^{q}\right)-c^{\alpha}\left(\frac{b-a}{4}\right)^{2 \alpha} \frac{B_{\alpha}(2,2)}{\Gamma(1+\alpha)}\right]^{\frac{1}{q}} \\
+\left[\frac{\Gamma(1+\alpha)}{\Gamma(1+2 \alpha)}\left(\left|f^{(2 \alpha)}\left(\frac{a+3 b}{4}\right)\right|^{q}+\left|f^{(2 \alpha)}\left(\frac{a+b}{2}\right)\right|^{q}\right)-c^{\alpha}\left(\frac{b-a}{4}\right)^{2 \alpha} \frac{B_{\alpha}(2,2)}{\Gamma(1+\alpha)}\right]^{\frac{1}{q}} \\
\left.+\sqrt[q]{\frac{\Gamma(1+\alpha)}{\Gamma(1+2 \alpha)}\left(\left|f^{(2 \alpha)}(b)\right|^{q}+\left|f^{(2 \alpha)}\left(\frac{a+3 b}{4}\right)\right|^{q}\right)-c^{\alpha}\left(\frac{b-a}{4}\right)^{2 \alpha} \frac{B_{\alpha}(2,2)}{\Gamma(1+\alpha)}}\right\} .
\end{gathered}
$$


Proof. From Lemmas 1,2 and 3 Definition 2.2 generalized strongly convexity of $\left|f^{(2 \alpha)}\right|^{q}$ and properties of the modulus, we have

$$
\begin{aligned}
& \left|A_{f}^{(\alpha)}(a, b)\right| \leq \frac{(b-a)^{2 \alpha}}{4^{3 \alpha} \Gamma(1+\alpha) \Gamma(1+2 \alpha)} \\
& \times \int_{0}^{1} t^{2 \alpha}\left[\left|f^{(2 \alpha)}\left(\left(\frac{3 a+b}{4}\right) t+(1-t) a\right)\right|+\left|f^{(2 \alpha)}\left(\left(\frac{a+b}{2}\right) t+\left(\frac{3 a+b}{4}\right)(1-t)\right)\right|\right. \\
& \left.+\left|f^{(2 \alpha)}\left(\left(\frac{a+3 b}{4}\right) t+\left(\frac{a+b}{2}\right)(1-t)\right)\right|+\left|f^{(2 \alpha)}\left(b t+\left(\frac{a+3 b}{4}\right)(1-t)\right)\right|\right](d t)^{\alpha} \\
& \leq \frac{(b-a)^{2 \alpha}}{4^{3 \alpha} \Gamma(1+2 \alpha)}\left(\frac{1}{\Gamma(1+\alpha)} \int_{0}^{1} t^{2 p \alpha}(d t)^{\alpha}\right)^{\frac{1}{p}} \\
& \times\left\{\left(\frac{1}{\Gamma(1+\alpha)} \int_{0}^{1}\left|f^{(2 \alpha)}\left(\left(\frac{3 a+b}{4}\right) t+(1-t) a\right)\right|^{q}(d t)^{\alpha}\right)^{\frac{1}{q}}\right. \\
& +\left(\frac{1}{\Gamma(1+\alpha)} \int_{0}^{1}\left|f^{(2 \alpha)}\left(\left(\frac{a+b}{2}\right) t+\left(\frac{3 a+b}{4}\right)(1-t)\right)\right|^{q}(d t)^{\alpha}\right)^{\frac{1}{q}} \\
& +\left(\frac{1}{\Gamma(1+\alpha)} \int_{0}^{1}\left|f^{(2 \alpha)}\left(\left(\frac{a+3 b}{4}\right) t+\left(\frac{a+b}{2}\right)(1-t)\right)\right|^{q}(d t)^{\alpha}\right)^{\frac{1}{q}} \\
& \left.+\left(\frac{1}{\Gamma(1+\alpha)} \int_{0}^{1}\left|f^{(2 \alpha)}\left(b t+\left(\frac{a+3 b}{4}\right)(1-t)\right)\right|^{q}(d t)^{\alpha}\right)^{\frac{1}{q}}\right\} \\
& \leq \frac{(b-a)^{2 \alpha}}{4^{3 \alpha} \Gamma(1+2 \alpha)} \sqrt[p]{\frac{\Gamma(1+2 p \alpha)}{\Gamma(1+(2 p+1) \alpha)}} \\
& \times\left\{\left[\frac { 1 } { \Gamma ( 1 + \alpha ) } \int _ { 0 } ^ { 1 } \left(t^{\alpha}\left|f^{(2 \alpha)}\left(\frac{3 a+b}{4}\right)\right|^{q}+(1-t)^{\alpha}\left|f^{(2 \alpha)}(a)\right|^{q}\right.\right.\right. \\
& \left.\left.-c^{\alpha} t^{\alpha}(1-t)^{\alpha}\left(\frac{3 a+b}{4}-a\right)^{2 \alpha}\right)(d t)^{\alpha}\right]^{\frac{1}{q}} \\
& +\left[\frac { 1 } { \Gamma ( 1 + \alpha ) } \int _ { 0 } ^ { 1 } \left(t^{\alpha}\left|f^{(2 \alpha)}\left(\frac{a+b}{2}\right)\right|^{q}+(1-t)^{\alpha}\left|f^{(2 \alpha)}\left(\frac{3 a+b}{4}\right)\right|^{q}\right.\right. \\
& \left.\left.-c^{\alpha} t^{\alpha}(1-t)^{\alpha}\left(\frac{a+b}{2}-\frac{3 a+b}{4}\right)^{2 \alpha}\right)(d t)^{\alpha}\right]^{\frac{1}{q}} \\
& +\left[\frac { 1 } { \Gamma ( 1 + \alpha ) } \int _ { 0 } ^ { 1 } \left(t^{\alpha}\left|f^{(2 \alpha)}\left(\frac{a+3 b}{4}\right)\right|^{q}+(1-t)^{\alpha}\left|f^{(2 \alpha)}\left(\frac{a+b}{2}\right)\right|^{q}\right.\right. \\
& \left.\left.-c^{\alpha} t^{\alpha}(1-t)^{\alpha}\left(\frac{a+3 b}{4}-\frac{a+b}{2}\right)^{2 \alpha}\right)(d t)^{\alpha}\right]^{\frac{1}{q}} \\
& +\left[\frac { 1 } { \Gamma ( 1 + \alpha ) } \int _ { 0 } ^ { 1 } \left(t^{\alpha}\left|f^{(2 \alpha)}(b)\right|^{q}+(1-t)^{\alpha}\left|f^{(2 \alpha)}\left(\frac{a+3 b}{4}\right)\right|^{q}\right.\right. \\
& \left.\left.\left.-c^{\alpha} t^{\alpha}(1-t)^{\alpha}\left(b-\frac{a+3 b}{4}\right)^{2 \alpha}\right)(d t)^{\alpha}\right]^{\frac{1}{q}}\right\}
\end{aligned}
$$




$$
\begin{aligned}
& =\frac{(b-a)^{2 \alpha}}{4^{3 \alpha} \Gamma(1+2 \alpha)} \sqrt[p]{\frac{\Gamma(1+2 p \alpha)}{\Gamma(1+(2 p+1) \alpha)}} \\
& \times\left\{\sqrt[q]{\frac{\Gamma(1+\alpha)}{\Gamma(1+2 \alpha)}\left(\left|f^{(2 \alpha)}\left(\frac{3 a+b}{4}\right)\right|^{q}+\left|f^{(2 \alpha)}(a)\right|^{q}\right)-c^{\alpha}\left(\frac{b-a}{4}\right)^{2 \alpha} \frac{B_{\alpha}(2,2)}{\Gamma(1+\alpha)}}\right. \\
& +\left[\frac{\Gamma(1+\alpha)}{\Gamma(1+2 \alpha)}\left(\left|f^{(2 \alpha)}\left(\frac{a+b}{2}\right)\right|^{q}+\left|f^{(2 \alpha)}\left(\frac{3 a+b}{4}\right)\right|^{q}\right)-c^{\alpha}\left(\frac{b-a}{4}\right)^{2 \alpha} \frac{B_{\alpha}(2,2)}{\Gamma(1+\alpha)}\right]^{\frac{1}{q}} \\
& +\left[\frac{\Gamma(1+\alpha)}{\Gamma(1+2 \alpha)}\left(\left|f^{(2 \alpha)}\left(\frac{a+3 b}{4}\right)\right|^{q}+\left|f^{(2 \alpha)}\left(\frac{a+b}{2}\right)\right|^{q}\right)-c^{\alpha}\left(\frac{b-a}{4}\right)^{2 \alpha} \frac{B_{\alpha}(2,2)}{\Gamma(1+\alpha)}\right]^{\frac{1}{q}} \\
& \left.+\sqrt[q]{\frac{\Gamma(1+\alpha)}{\Gamma(1+2 \alpha)}\left(\left|f^{(2 \alpha)}(b)\right|^{q}+\left|f^{(2 \alpha)}\left(\frac{a+3 b}{4}\right)\right|^{q}\right)-c^{\alpha}\left(\frac{b-a}{4}\right)^{2 \alpha} \frac{B_{\alpha}(2,2)}{\Gamma(1+\alpha)}}\right\} .
\end{aligned}
$$

So, the proof of this theorem is completed.

We point out some special cases of Theorem 5 .

Corollary 5.1. Under assumptions of Theorem 5, if we choose $p=q=2$, we get the following generalized trapezium-like inequality:

$$
\begin{gathered}
\left|A_{f}^{(\alpha)}(a, b)\right| \leq \frac{(b-a)^{2 \alpha}}{4^{3 \alpha} \Gamma(1+2 \alpha)} \sqrt{\frac{\Gamma(1+4 \alpha)}{\Gamma(1+5 \alpha)}} \\
\times\left\{\sqrt{\frac{\Gamma(1+\alpha)}{\Gamma(1+2 \alpha)}\left(\left|f^{(2 \alpha)}\left(\frac{3 a+b}{4}\right)\right|^{2}+\left|f^{(2 \alpha)}(a)\right|^{2}\right)-c^{\alpha}\left(\frac{b-a}{4}\right)^{2 \alpha} \frac{B_{\alpha}(2,2)}{\Gamma(1+\alpha)}}\right. \\
+\left[\frac{\Gamma(1+\alpha)}{\Gamma(1+2 \alpha)}\left(\left|f^{(2 \alpha)}\left(\frac{a+b}{2}\right)\right|^{2}+\left|f^{(2 \alpha)}\left(\frac{3 a+b}{4}\right)\right|^{2}\right)-c^{\alpha}\left(\frac{b-a}{4}\right)^{2 \alpha} \frac{B_{\alpha}(2,2)}{\Gamma(1+\alpha)}\right]^{\frac{1}{2}} \\
+\left[\frac{\Gamma(1+\alpha)}{\Gamma(1+2 \alpha)}\left(\left|f^{(2 \alpha)}\left(\frac{a+3 b}{4}\right)\right|^{2}+\left|f^{(2 \alpha)}\left(\frac{a+b}{2}\right)\right|^{2}\right)-c^{\alpha}\left(\frac{b-a}{4}\right)^{2 \alpha} \frac{B_{\alpha}(2,2)}{\Gamma(1+\alpha)}\right]^{\frac{1}{2}} \\
\left.+\sqrt{\frac{\Gamma(1+\alpha)}{\Gamma(1+2 \alpha)}\left(\left|f^{(2 \alpha)}(b)\right|^{2}+\left|f^{(2 \alpha)}\left(\frac{a+3 b}{4}\right)\right|^{2}\right)-c^{\alpha}\left(\frac{b-a}{4}\right)^{2 \alpha} \frac{B_{\alpha}(2,2)}{\Gamma(1+\alpha)}}\right\} .
\end{gathered}
$$

Corollary 5.2. Under assumptions of Theorem 5, taking $c \rightarrow 0^{+}$, we get the following generalized trapezium-like inequality for generalized convex function:

$$
\begin{aligned}
& \left|A_{f}^{(\alpha)}(a, b)\right| \leq \frac{(b-a)^{2 \alpha}}{4^{3 \alpha} \Gamma(1+2 \alpha)} \sqrt[p]{\frac{\Gamma(1+2 p \alpha)}{\Gamma(1+(2 p+1) \alpha)}} \sqrt[q]{\frac{\Gamma(1+\alpha)}{\Gamma(1+2 \alpha)}} \\
& \times\left\{\sqrt[q]{\mid f^{\left.(2 \alpha)\left(\frac{3 a+b}{4}\right)\right|^{q}+\left|f^{(2 \alpha)}(a)\right|^{q}}}+\sqrt[q]{\left|f(2 \alpha)\left(\frac{a+b}{2}\right)\right|^{q}+\mid f^{\left.(2 \alpha)\left(\frac{3 a+b}{4}\right)\right|^{q}}}\right.
\end{aligned}
$$

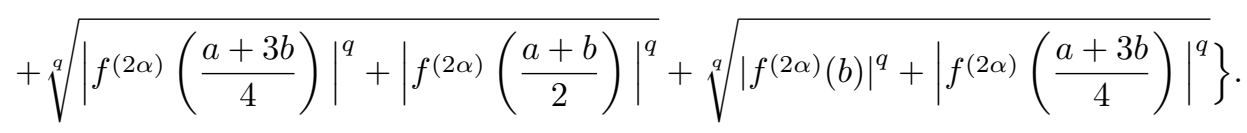


Corollary 5.3. Under assumptions of Theorem 5, by taking $K=\left\|f^{(2 \alpha)}\right\|_{\infty}$, we get the following generalized trapezium-like inequality:

$$
\begin{gathered}
\left|A_{f}^{(\alpha)}(a, b)\right| \leq \frac{(b-a)^{2 \alpha}}{4^{3 \alpha-1} \Gamma(1+2 \alpha)} \sqrt[p]{\frac{\Gamma(1+2 p \alpha)}{\Gamma(1+(2 p+1) \alpha)}} \\
\quad \times \sqrt[q]{\frac{\Gamma(1+\alpha)}{\Gamma(1+2 \alpha)} 2 K^{q}-c^{\alpha}\left(\frac{b-a}{4}\right)^{2 \alpha} \frac{B_{\alpha}(2,2)}{\Gamma(1+\alpha)}} .
\end{gathered}
$$

Corollary 5.4. Under assumptions of Corollary 5.3, taking $c \rightarrow 0^{+}$, we get the following generalized trapezium-like inequality for generalized convex function:

$$
\left|A_{f}^{(\alpha)}(a, b)\right| \leq \frac{K \sqrt[q]{2}(b-a)^{2 \alpha}}{4^{3 \alpha-1} \Gamma(1+2 \alpha)} \sqrt[p]{\frac{\Gamma(1+2 p \alpha)}{\Gamma(1+(2 p+1) \alpha)}} \sqrt[q]{\frac{\Gamma(1+\alpha)}{\Gamma(1+2 \alpha)}} .
$$

Theorem 6. Suppose that the assumptions of Lema 3 are satisfied. If $\left|f^{(2 \alpha)}\right|^{q}$ is generalized strongly convex, then for $q \geq 1$ and $c \in \Re^{+}$, the following inequality holds:

$$
\begin{aligned}
& \left|A_{f}^{(\alpha)}(a, b)\right| \leq \frac{(b-a)^{2 \alpha}}{4^{3 \alpha} \Gamma(1+2 \alpha)}\left(\frac{\Gamma(1+2 \alpha)}{\Gamma(1+3 \alpha)}\right)^{1-\frac{1}{q}} \\
& \times\left\{\sqrt[q]{\left|f^{(2 \alpha)}\left(\frac{3 a+b}{4}\right)\right|^{q} C(\alpha)+\left|f^{(2 \alpha)}(a)\right|^{q} D(\alpha)-c^{\alpha}\left(\frac{b-a}{4}\right)^{2 \alpha} \frac{B_{\alpha}(4,2)}{\Gamma(1+\alpha)}}\right. \\
& +\left[\left|f^{(2 \alpha)}\left(\frac{a+b}{2}\right)\right|^{q} C(\alpha)+\left|f^{(2 \alpha)}\left(\frac{3 a+b}{4}\right)\right|^{q} D(\alpha)-c^{\alpha}\left(\frac{b-a}{4}\right)^{2 \alpha} \frac{B_{\alpha}(4,2)}{\Gamma(1+\alpha)}\right]^{\frac{1}{q}} \\
& +\left[\left|f^{(2 \alpha)}\left(\frac{a+3 b}{4}\right)\right|^{q} C(\alpha)+\left|f^{(2 \alpha)}\left(\frac{a+b}{2}\right)\right|^{q} D(\alpha)-c^{\alpha}\left(\frac{b-a}{4}\right)^{2 \alpha} \frac{B_{\alpha}(4,2)}{\Gamma(1+\alpha)}\right]^{\frac{1}{q}} \\
& +\sqrt[q]{\left.\left|f^{(2 \alpha)}(b)\right|^{q} C(\alpha)+\left|f^{(2 \alpha)}\left(\frac{a+3 b}{4}\right)\right|^{q} D(\alpha)-c^{\alpha}\left(\frac{b-a}{4}\right)^{2 \alpha} \frac{B_{\alpha}(4,2)}{\Gamma(1+\alpha)}\right\}},
\end{aligned}
$$

where

$$
C(\alpha)=\frac{\Gamma(1+3 \alpha)}{\Gamma(1+4 \alpha)}, \quad D(\alpha)=\frac{\Gamma(1+\alpha)}{\Gamma(1+2 \alpha)}-2^{\alpha} \frac{\Gamma(1+2 \alpha)}{\Gamma(1+3 \alpha)}+C(\alpha) .
$$

Proof. From Lemmas 1 and 3 . Definition 2.2 generalized power-mean inequality, generalized strongly convexity of $\left|f^{(2 \alpha)}\right|^{q}$ and properties of the modulus, we have

$$
\begin{gathered}
\left|A_{f}^{(\alpha)}(a, b)\right| \leq \frac{(b-a)^{2 \alpha}}{4^{3 \alpha} \Gamma(1+\alpha) \Gamma(1+2 \alpha)} \\
\times \int_{0}^{1} t^{2 \alpha}\left[\left|f^{(2 \alpha)}\left(\left(\frac{3 a+b}{4}\right) t+(1-t) a\right)\right|+\left|f^{(2 \alpha)}\left(\left(\frac{a+b}{2}\right) t+\left(\frac{3 a+b}{4}\right)(1-t)\right)\right|\right. \\
\left.+\left|f^{(2 \alpha)}\left(\left(\frac{a+3 b}{4}\right) t+\left(\frac{a+b}{2}\right)(1-t)\right)\right|+\left|f^{(2 \alpha)}\left(b t+\left(\frac{a+3 b}{4}\right)(1-t)\right)\right|\right](d t)^{\alpha}
\end{gathered}
$$




$$
\begin{aligned}
& \leq \frac{(b-a)^{2 \alpha}}{4^{3 \alpha} \Gamma(1+2 \alpha)}\left(\frac{1}{\Gamma(1+\alpha)} \int_{0}^{1} t^{2 \alpha}(d t)^{\alpha}\right)^{1-\frac{1}{q}} \\
& \times\left\{\left(\frac{1}{\Gamma(1+\alpha)} \int_{0}^{1} t^{2 \alpha}\left|f^{(2 \alpha)}\left(\left(\frac{3 a+b}{4}\right) t+(1-t) a\right)\right|^{q}(d t)^{\alpha}\right)^{\frac{1}{q}}\right. \\
& +\left(\frac{1}{\Gamma(1+\alpha)} \int_{0}^{1} t^{2 \alpha}\left|f^{(2 \alpha)}\left(\left(\frac{a+b}{2}\right) t+\left(\frac{3 a+b}{4}\right)(1-t)\right)\right|^{q}(d t)^{\alpha}\right)^{\frac{1}{q}} \\
& +\left(\frac{1}{\Gamma(1+\alpha)} \int_{0}^{1} t^{2 \alpha}\left|f^{(2 \alpha)}\left(\left(\frac{a+3 b}{4}\right) t+\left(\frac{a+b}{2}\right)(1-t)\right)\right|^{q}(d t)^{\alpha}\right)^{\frac{1}{q}} \\
& \left.+\left(\frac{1}{\Gamma(1+\alpha)} \int_{0}^{1} t^{2 \alpha}\left|f^{(2 \alpha)}\left(b t+\left(\frac{a+3 b}{4}\right)(1-t)\right)\right|^{q}(d t)^{\alpha}\right)^{\frac{1}{q}}\right\} \\
& \leq \frac{(b-a)^{2 \alpha}}{4^{3 \alpha} \Gamma(1+2 \alpha)}\left(\frac{\Gamma(1+2 \alpha)}{\Gamma(1+3 \alpha)}\right)^{1-\frac{1}{q}} \\
& \times\left\{\left[\frac { 1 } { \Gamma ( 1 + \alpha ) } \int _ { 0 } ^ { 1 } t ^ { 2 \alpha } \left(t^{\alpha}\left|f^{(2 \alpha)}\left(\frac{3 a+b}{4}\right)\right|^{q}+(1-t)^{\alpha}\left|f^{(2 \alpha)}(a)\right|^{q}\right.\right.\right. \\
& \left.\left.-c^{\alpha} t^{\alpha}(1-t)^{\alpha}\left(\frac{3 a+b}{4}-a\right)^{2 \alpha}\right)(d t)^{\alpha}\right]^{\frac{1}{q}} \\
& +\left[\frac { 1 } { \Gamma ( 1 + \alpha ) } \int _ { 0 } ^ { 1 } t ^ { 2 \alpha } \left(t^{\alpha}\left|f^{(2 \alpha)}\left(\frac{a+b}{2}\right)\right|^{q}+(1-t)^{\alpha}\left|f^{(2 \alpha)}\left(\frac{3 a+b}{4}\right)\right|^{q}\right.\right. \\
& \left.\left.-c^{\alpha} t^{\alpha}(1-t)^{\alpha}\left(\frac{a+b}{2}-\frac{3 a+b}{4}\right)^{2 \alpha}\right)(d t)^{\alpha}\right]^{\frac{1}{q}} \\
& +\left[\frac { 1 } { \Gamma ( 1 + \alpha ) } \int _ { 0 } ^ { 1 } t ^ { 2 \alpha } \left(t^{\alpha}\left|f^{(2 \alpha)}\left(\frac{a+3 b}{4}\right)\right|^{q}+(1-t)^{\alpha}\left|f^{(2 \alpha)}\left(\frac{a+b}{2}\right)\right|^{q}\right.\right. \\
& \left.\left.-c^{\alpha} t^{\alpha}(1-t)^{\alpha}\left(\frac{a+3 b}{4}-\frac{a+b}{2}\right)^{2 \alpha}\right)(d t)^{\alpha}\right]^{\frac{1}{q}} \\
& +\left[\frac { 1 } { \Gamma ( 1 + \alpha ) } \int _ { 0 } ^ { 1 } t ^ { 2 \alpha } \left(t^{\alpha}\left|f^{(2 \alpha)}(b)\right|^{q}+(1-t)^{\alpha}\left|f^{(2 \alpha)}\left(\frac{a+3 b}{4}\right)\right|^{q}\right.\right. \\
& \left.\left.\left.-c^{\alpha} t^{\alpha}(1-t)^{\alpha}\left(b-\frac{a+3 b}{4}\right)^{2 \alpha}\right)(d t)^{\alpha}\right]^{\frac{1}{q}}\right\} \\
& =\frac{(b-a)^{2 \alpha}}{4^{3 \alpha} \Gamma(1+2 \alpha)}\left(\frac{\Gamma(1+2 \alpha)}{\Gamma(1+3 \alpha)}\right)^{1-\frac{1}{q}} \\
& \times\left\{\sqrt[q]{\left|f^{(2 \alpha)}\left(\frac{3 a+b}{4}\right)\right|^{q} C(\alpha)+\left|f^{(2 \alpha)}(a)\right|^{q} D(\alpha)-c^{\alpha}\left(\frac{b-a}{4}\right)^{2 \alpha} \frac{B_{\alpha}(4,2)}{\Gamma(1+\alpha)}}\right. \\
& +\left[\left.\left|f^{(2 \alpha)}\left(\frac{a+b}{2}\right)\right|\right|^{q} C(\alpha)+\left|f^{(2 \alpha)}\left(\frac{3 a+b}{4}\right)\right|^{q} D(\alpha)-c^{\alpha}\left(\frac{b-a}{4}\right)^{2 \alpha} \frac{B_{\alpha}(4,2)}{\Gamma(1+\alpha)}\right]^{\frac{1}{q}} \\
& +\left[\left|f^{(2 \alpha)}\left(\frac{a+3 b}{4}\right)\right|^{q} C(\alpha)+\left|f^{(2 \alpha)}\left(\frac{a+b}{2}\right)\right|^{q} D(\alpha)-c^{\alpha}\left(\frac{b-a}{4}\right)^{2 \alpha} \frac{B_{\alpha}(4,2)}{\Gamma(1+\alpha)}\right]^{\frac{1}{q}}
\end{aligned}
$$




$$
+\sqrt[q]{\left.\left|f^{(2 \alpha)}(b)\right|{ }^{q} C(\alpha)+\left|f^{(2 \alpha)}\left(\frac{a+3 b}{4}\right)\right|^{q} D(\alpha)-c^{\alpha}\left(\frac{b-a}{4}\right)^{2 \alpha} \frac{B_{\alpha}(4,2)}{\Gamma(1+\alpha)}\right\}} .
$$

So, the proof of this theorem is completed.

We point out some special cases of Theorem 6 .

Corollary 6.1. Under assumptions of Theorem 6 , if we choose $q=1$, we get the following generalized trapezium-like inequality:

$$
\begin{gathered}
\left|A_{f}^{(\alpha)}(a, b)\right| \leq \frac{(b-a)^{2 \alpha}}{4^{3 \alpha} \Gamma(1+2 \alpha)} \\
\times\left\{\left|f^{(2 \alpha)}\left(\frac{3 a+b}{4}\right)\right| C(\alpha)+\left|f^{(2 \alpha)}(a)\right| D(\alpha)-c^{\alpha}\left(\frac{b-a}{4}\right)^{2 \alpha} \frac{B_{\alpha}(4,2)}{\Gamma(1+\alpha)}\right. \\
+\left|f^{(2 \alpha)}\left(\frac{a+b}{2}\right)\right| C(\alpha)+\left|f^{(2 \alpha)}\left(\frac{3 a+b}{4}\right)\right| D(\alpha)-c^{\alpha}\left(\frac{b-a}{4}\right)^{2 \alpha} \frac{B_{\alpha}(4,2)}{\Gamma(1+\alpha)} \\
+\left|f^{(2 \alpha)}\left(\frac{a+3 b}{4}\right)\right| C(\alpha)+\left|f^{(2 \alpha)}\left(\frac{a+b}{2}\right)\right| D(\alpha)-c^{\alpha}\left(\frac{b-a}{4}\right)^{2 \alpha} \frac{B_{\alpha}(4,2)}{\Gamma(1+\alpha)} \\
\left.+\left|f^{(2 \alpha)}(b)\right| C(\alpha)+\left|f^{(2 \alpha)}\left(\frac{a+3 b}{4}\right)\right| D(\alpha)-c^{\alpha}\left(\frac{b-a}{4}\right)^{2 \alpha} \frac{B_{\alpha}(4,2)}{\Gamma(1+\alpha)}\right\} .
\end{gathered}
$$

Corollary 6.2. Under assumptions of Theorem 6 , taking $c \rightarrow 0^{+}$, we get the following generalized trapezium-like inequality for generalized convex function:

$$
\begin{aligned}
& \left|A_{f}^{(\alpha)}(a, b)\right| \leq \frac{(b-a)^{2 \alpha}}{4^{3 \alpha} \Gamma(1+2 \alpha)}\left(\frac{\Gamma(1+2 \alpha)}{\Gamma(1+3 \alpha)}\right)^{1-\frac{1}{q}} \\
& \times\left\{\sqrt[q]{\mid f^{\left.(2 \alpha)\left(\frac{3 a+b}{4}\right)\right|^{q} C(\alpha)+\left|f^{(2 \alpha)}(a)\right|^{q} D(\alpha)}}\right. \\
& +\sqrt[q]{\left|f^{(2 \alpha)}\left(\frac{a+b}{2}\right)\right|^{q} C(\alpha)+\left|f^{(2 \alpha)}\left(\frac{3 a+b}{4}\right)\right|^{q} D(\alpha)}
\end{aligned}
$$

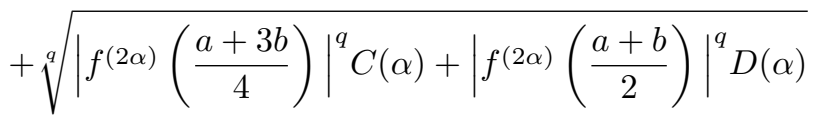

$$
\begin{aligned}
& +\sqrt[q]{\left.\left|f^{(2 \alpha)}(b)\right|^{q} C(\alpha)+\left|f^{(2 \alpha)}\left(\frac{a+3 b}{4}\right)\right|^{q} D(\alpha)\right\}} .
\end{aligned}
$$

Corollary 6.3. Under assumptions of Theorem 6 , by taking $K=\left\|f^{(2 \alpha)}\right\|_{\infty}$, we get the following generalized trapezium-like inequality:

$$
\begin{aligned}
& \left|A_{f}^{(\alpha)}(a, b)\right| \leq \frac{(b-a)^{2 \alpha}}{4^{3 \alpha-1} \Gamma(1+2 \alpha)}\left(\frac{\Gamma(1+2 \alpha)}{\Gamma(1+3 \alpha)}\right)^{1-\frac{1}{q}} \\
& \times \sqrt[q]{K^{q}[D(\alpha)+C(\alpha)]-c^{\alpha}\left(\frac{b-a}{4}\right)^{2 \alpha} \frac{B_{\alpha}(4,2)}{\Gamma(1+\alpha)}} .
\end{aligned}
$$


Corollary 6.4. Under assumptions of Corollary 6.3, taking $c \rightarrow 0^{+}$, we get the following generalized trapezium-like inequality for generalized convex function:

$$
\left|A_{f}^{(\alpha)}(a, b)\right| \leq \frac{K \sqrt[q]{D(\alpha)+C(\alpha)}(b-a)^{2 \alpha}}{4^{3 \alpha-1} \Gamma(1+2 \alpha)}\left(\frac{\Gamma(1+2 \alpha)}{\Gamma(1+3 \alpha)}\right)^{1-\frac{1}{q}} .
$$

Remark 2.2: For $\alpha=1$, by our Theorems 5 and 6, we can obtain some new estimates on generalization of trapezium-like inequalities for twice differentiable mappings that are in absolute value at certain powers strongly convex.

\section{REFERENCES}

[1] P. Agarwal, Some inequalities involving Hadamard type $k$-fractional integral operators, Math. Methods Appl. Sci., 40 (2017), 3882-3891.

[2] P. Agarwal, M. Jleli, M. Toar, Certain Hermite-Hadamard type inequalities via generalized $k$-fractional integrals, J. Inequal. Appl., 2017 (2017), pp. 10.

[3] G. Anastassiou, Fractional Differentiation Inequalities, Springer, NY, Heidelberg, 2009.

[4] G. Anastassiou, Advances on Fractional Inequalities, Springer, NY, 2011.

[5] G. Anastassiou, Intelligent Mathematics: Computational Analysis, Springer, Heidelberg, New York, 2011.

[6] G. Anastassiou, A. Kashuri, R. Liko, Local fractional integrals involving generalized strongly m-convex appings, Arab. J. Math., 2018.

[7] H. Budak, M. Z. Sarikaya, H. Yildirim, New inequalities for local fractional integrals, Iran J Sci Technol Trans Sci, (2017), pp. 8.

[8] J. S. Choi, E. Set, M. Toar, Certain generalized Ostrowski type inequalities for local fractional integrals, Commun. Korean Math. Soc., 32 (2017), 601-617.

[9] S. Erden, M. Z. Sarikaya, Generalized Pompeiu type inequalities for local fractional integrals and its applications, Appl. Math. Comput., 274, (2016), 282-291.

[10] G. Juarie, Table of some basic fractional calculus formulae derived from a modified Riemann-Liouville derivative for non-differentiable functions, Appl. Math. Lett., 22 (2009), 378-385.

[11] T. Lara, On strongly m-convex functions, Math. Aeterna, 5(3) (2015), 521-535.

[12] H. X. Mo, Generalized Hermite-Hadamard inequalities involving local fractional integral, Arxiv, 2014 (2014), pp. 8.

[13] H. X. Mo, X. Sui, Generalized s-convex functions on fractal sets, Abstr. Appl. Anal., 2014 (2014), pp. 8.

[14] H. X. Mo, X. Sui, Hermite-Hadamard type inequalities for generalized s-convex functions on real linear fractal set $\mathbb{R}^{\alpha}(0<\alpha \leq 1)$, Math. Sci. (Springer), 11 (2017), 241-246.

[15] H. X. Mo, X. Sui, D. Y. Yu, Generalized convex functions on fractal sets and two related inequalities, Abstr. Appl. Anal., 2014 (2014), pp. 7.

[16] M. Z. Sarikaya, H. Budak, Generalized Ostrowski type inequalities for local fractional integrals, Proc. Amer. Math. Soc., 145 (2017), 1527-1538.

[17] M. Z. Sarikaya, S. Erden, H. Budak, Some generalized Ostrowski type inequalities involving local fractional integrals and applications, RGMIA Research Report Collection, 18 (2015), pp. 12.

[18] M. Z. Sarikaya, M. Tunç, H. Budak, On generalized some integral inequalities for local fractional integrals, Appl. Math. Comput., 276 (2016), 316-323.

[19] E. Set, M. Tomar, New inequalities of Hermite-Hadamard type for generalized convex functions with applications, Facta Univ. Ser. Math. Inform., 31 (2016), 383-397.

[20] H. M. Srivastava, J. S. Choi, Zeta and q-Zeta functions and associated series and integrals, Elsevier, Inc., Amsterdam, 2012. 
[21] M. Tomar, P. Agarwal, M. Jleli, B. Samet, Certain Ostrowski type inequalities for generalized s-convex functions, J. Nonlinear Sci. Appl., 10 (2017), 5947-5957.

[22] X. J. Yang, Generalized local fractional Taylor's formula with local fractional derivative, Arxiv, 2011 (2011), pp. 5.

[23] X. J. Yang, Local fractional functional analysis and its applications, Asian Academic publisher Limited, Hong Kong, 2011.

[24] X. J. Yang, Advanced local fractional calculus and its applications, World Science Publisher, New York, 2012.

[25] X. J. Yang, Local fractional Fourier analysis, Adv. Mech. Eng. Appl., 1 (2012), 12-16.

[26] X. J. Yang, Local fractional integral equations and their applications, Adv. Comput. Sci. Appl., 1 (2012), 234-239.

[27] Y. J. Yang, D. Baleanu, X. J. Yang, Analysis of fractal wave equations by local fractional Fourier series method, Adv. Math. Phys., 2013 (2013), pp. 6.

1 Department of Mathematics, Faculty of Technical Science, University Ismail Qemali, Vlora, Albania

Email address: artionkashuri@gmail.com

2 Department of Mathematics, Faculty of Science and Arts, Düzce University, Düzce, Turkey

Email address: sarikayamz@gmail.com

Received 10.6.2020

Revised 1.10.2020

Accepted 1.10.2020 